\title{
Android application for ocular training in amblyopia
}

\author{
Daniela Mariana Barbu $^{1, *}$ \\ ${ }^{1}$ Transilvania University of Brasov, 29 Eroilor Bd., 500036 Brasov, Romania
}

\begin{abstract}
This paper propose a mobile application that runs on the Android system, an app that aims to help persons with amblyopia by running recovery exercises in a very simple and convenient way, right on their own phone. It is very important to know that training the amblyopic eye even five minutes a day can result in vision acuity recovery, so it is recommended to use this application every day. Moreover, it is very easy to use, with an intriguing and intuitive menu with very simple commands. The subject with amblyopia can do the exercises anywhere, anytime. Another advantage is the accessibility of the application, which is used by the phone, can be used at any time of the day; the user has to give only five minutes of his daily activities.
\end{abstract}

\section{Introduction}

The visual analyser is effectively built to provide optimal visual function. The light penetrates the eyes, crosses the transparent and refractive media, formed by cornea, aqueous, crystalline and vitreous, eventually reaching the retina where the image is formed [1]. The amount of light that enters the eye is regulated by the pupillary opening [2]. In the emetropic eye, transparent and refractive media contribute to the convergence of luminous rays and their focusing on the retina. It is a selective receptor for electromagnetic radiation between 375 and $760 \mathrm{~nm}$ [3]. Its photoreceptors are the ones that convert electricity into light energy, thanks to photo pigments that contain a protein role, rhodopsin [4]. On the other hand, the eyeball has the property of orienting the visual axis in the direction of the object fixed by the extrinsic muscles that rotate it around it. The muscles are commanded by oculomotor nerves so that the two eyes act simultaneously as one eye to achieve binocular and stereoscopic vision [5].

Amblyopia is the decrease in monocular visual acuity (measured with the best optical correction) undetermined by apparent organic causes of the posterior eye or the visual pathways. After Cernea [6] amblyopia is the diminution of visual acuity in the absence of visible organic lesions or organic lesions that do not adequately explain the decrease in vision. Vancea et al. [7] define amblyopia as a decrease in visual acuity having as a substrate the primary or secondary damage to neuronal circuits. Amblyopia is an alteration of the central area of the visual field, while its peripheral area is usually unaffected [3]. The more amblyopia appears earlier in life (interfering with the process of developing normal binocular vision), the worse it is. To the same extent, the earlier it is treated, the better the prognosis treatment. Yet, recovery exercises for amblyopic eye are recommended at any age at least to stop the degree of amblyopia already installed [5].

\section{The mobile application Amblioptix}

During this age of technology becomes increasingly shorter and are more and more work to be done, we divide between professional and personal life. Fortunately, technology makes stunning progress and gives modern man the means to make things much faster in the online environment that would otherwise have consumed him for quite some time. One of the most interesting aspects of technology development is the ability to intuitively relate to the wishes and needs of users, enabling them to optimize daily activities [8].

Now, according [9] the challenge for software developers is to create the smartest programs that mimic the human mind in terms of functioning, perform more and more complex tasks, and even self-optimize, similar to the process by which people constantly learn and memorizes new information every day.

Android is a software platform and operating system for mobile devices and phones, based on the Linux kernel originally developed by Google, and later by the Open Handset Alliance commercial consortium [10]. Android allows developers to write managed code in the Java programming language, controlling the device through the Java libraries developed by Google [11].

Amblioptix is a mobile application, developed with student Alexandru Spătaru, running only on the Android system, an application that aims to help persons with amblyopia by running recovery exercises in a very simple and convenient way, even on their own phone. It is very important to know that training of the amblyopic eye even for only five minutes a day can result in its disambiguation, so it is recommended to use this application every day. Moreover, it is very easy to use, with an attractive and intuitive menu with very simple controls. The subject with amblyopia can do the exercises anywhere, anytime. Another plus is the accessibility of the

\footnotetext{
* Corresponding author: dbarbu@unitbv.ro
} 
application, which is used by the phone, it can be used at any time of the day, and the user has to give only five minutes of his daily activities.

Table 1. Minimum phone requirements

\begin{tabular}{|l|l|}
\hline Requirement & Description \\
\hline Android version & 4.4 .2 or later \\
\hline Phone processor & Quad Core Processor, $1.0 \mathrm{GHz}$ \\
\hline RAM Memory & $500 \mathrm{MB}$ minimum \\
\hline Memory space & $4.25 \mathrm{MB}$ \\
\hline Screen resolution & $480 \times 800$ minimum \\
\hline
\end{tabular}

\subsection{Application design}

The application was developed in the Android Studio 2.3.3 development environment by using the Java and XML programming language, which is an extensible mark-up system, that is, it is simpler, a mark-up system similar to HTML, but it is much better and more dynamic, the key difference being that tags are not defined, the programmer being free to experiment. [12]

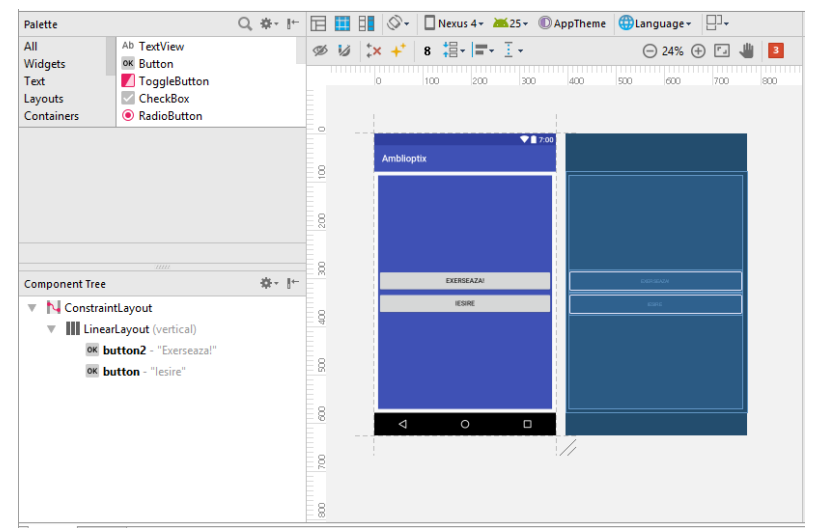

"Exit" button

public void onIesireBtnClick(View view) \{finish(); \}

"Practice" button

public void onExerseazaBtnClick (View view)

Intent myIntent $=$ new Intent (this, MeniuExercitii.class); startActivity (myIntent);

Fig. 1. Android Studio presentation

To begin with, the main menu of the application was created, where the two buttons, "Exersează" (Exercise) and "Ieșire" (Exit), intuitive names for their use, was introduced. Behind each button there is a method represented by a piece of code that gives meaning and representation in the application. For example, the "Ieșire" button will close the application, represented simply by finishing it, and the "Exersează" button will also open a new menu consisting of the actual recovery exercises.

By navigating through the exercise menu, you can find out how each button for each exercise has been implemented (Figure 2).

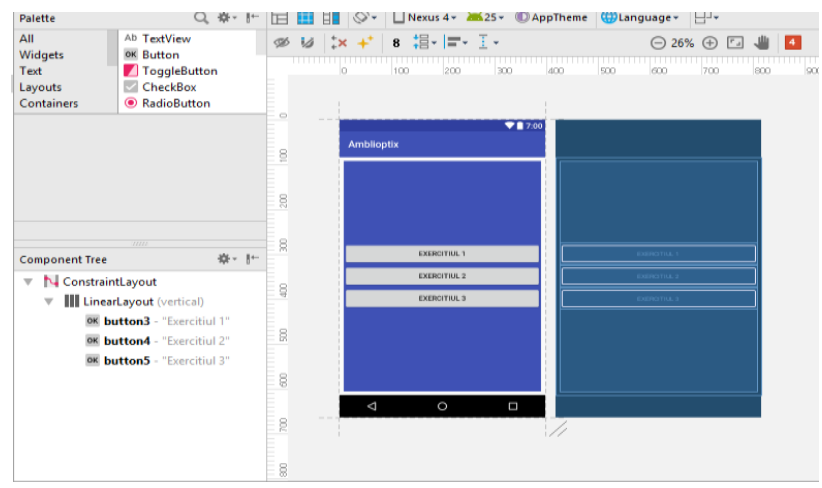

public void onExercitiul1BtnClick (View view) \{ Intent myIntent $=$ new Intent (this, Game.class); myIntent.putExtra("type", Exercitii.EXE1); startActivity (myIntent);

public void onExercitiul2BtnClick (View view) \{ Intent myIntent $=$ new Intent (this, Game.class); myIntent.putExtra("type", Exercitii.EXE2); startActivity (myIntent);

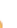

public void bnExercitiul3BtnClick(View view) Intent myIntent $=$ new Intent (this, Game.class); myIntent.putExtra("type", Exercitii.EXE3); startActivity (myIntent);

Fig. 2. Recovery exercises in Android Studio

Also, behind each exercise button were written recovery methods, which are designed to start the recovery protocol as efficiently as possible.

\subsection{Amblioptix implementation}

The application consists of two menus: principal menu (Figure 3) and exercises menu (Figure 4).

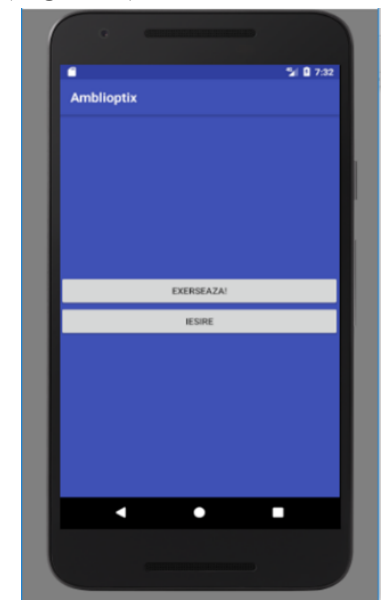

Fig. 3. Main menu

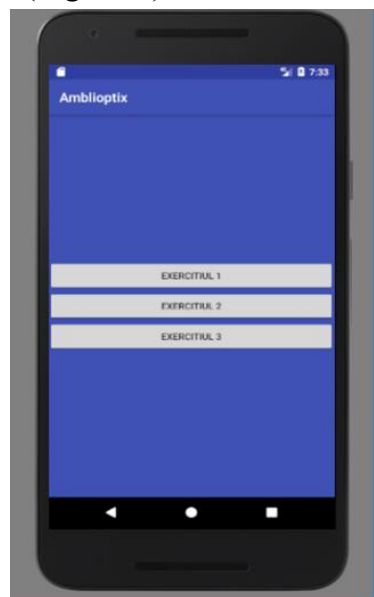

Fig. 4. Exercises menu
The "Exersează" button will send us to another menu where we will retrieve the recovery exercises, while the "Ieșire" button will result in closing the application in a simple and direct way.

All three exercises have the role of strengthening the external muscles of the eyeball along with the effective transmission of visual impulses from the retina to the visual cortex.

The use of this application is especially for people with amblyopia, and in order to have a remarkable result, it is necessary to strictly observe a daily program of use. 


\section{Exercise 1}

By accessing the first exercise of this application, one can see the black-and-white background (like chess table) and a red dot, a point that must always be watched by the amblyopic eye, the congener eye being in occlusion all this time.

In this exercise, the subject follows the red point, which carries a circular motion whose radius decreases to a certain limit, and then returns to its original value. Practically, the point moves initially to the inside, and then vice versa, returns to the shape of the original circle. This exercise can work for a maximum of five minutes, a value set in the program.
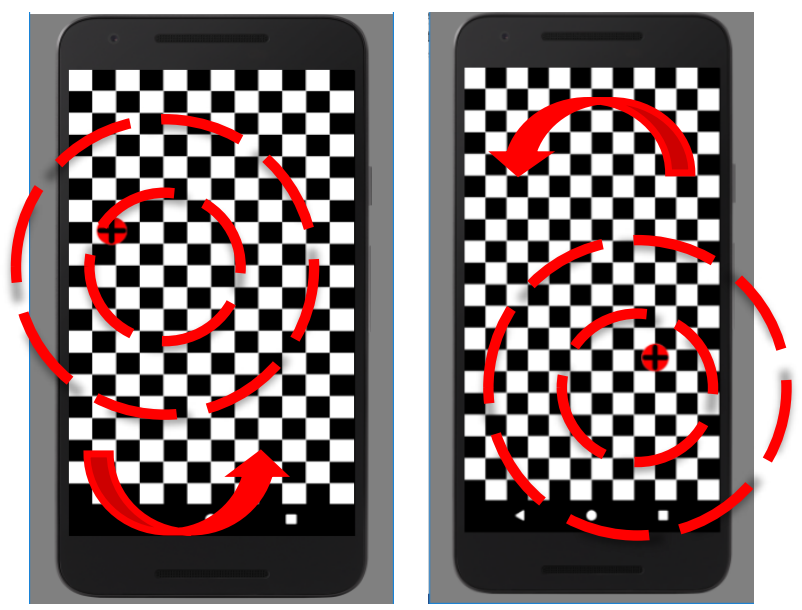

Fig. 5. Exercise No. 1

\section{Exercise 2}

Using the second exercise of this application, it can be notice the background represented by black and white background, and a red dot, a point that must always be watched by the amblyopic eye. In the second exercise, the subject strengthens the point, which, this time, intersects the two horizontal sides of the screen, following the motion of the point in the form of zigzag shapes (Figure 6). This exercise works for a period of five minutes, a value imposed by the program.
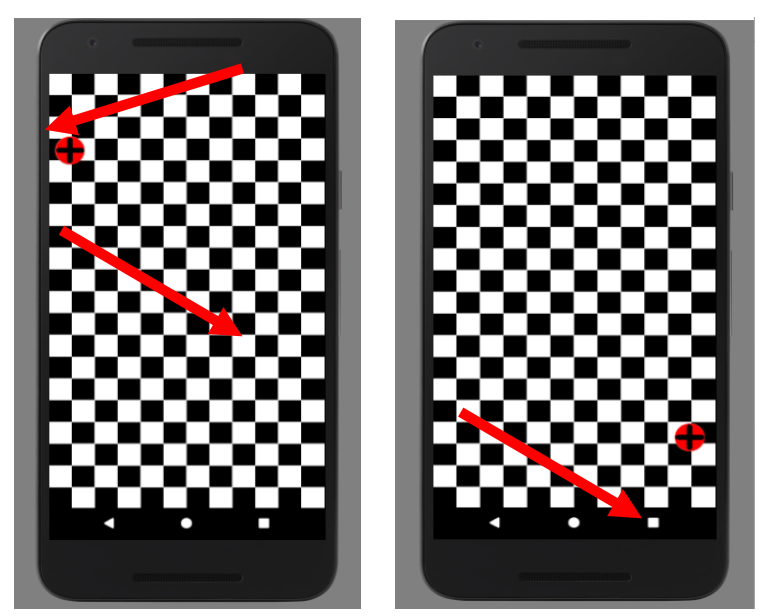

Fig. 6. Exercise No. 2

\section{Exercise 3}

By accessing the third exercise of this application, one can see the background in black and white, and the same red dot, a point that must always be watched by the amblyopic eye. This exercise is intended for sharper, more difficult to follow by the amblyopic eye, an exercise where the level of difficulty is high. The red dot rises to the vertical line of the screen until it touches the corner of the screen, and then suddenly changes its position by moving diagonally to the opposite corner of the screen (Figure 7). This movement is repeated over a period of five minutes, also a value imposed by the program.
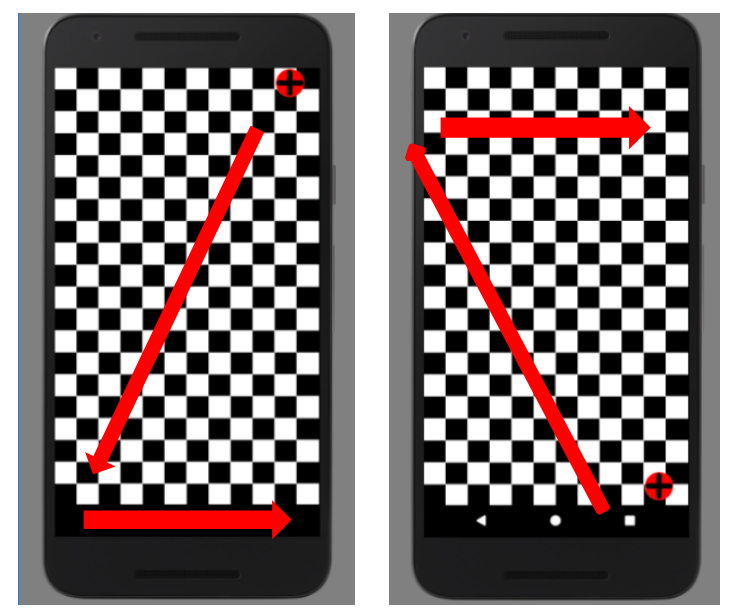

Fig. 7. Exercise No. 3

Instructions for using the application:

- The app installs on a phone whose operating system must be Android. Any version of it is supported for this application to run. After the application is installed, the Amblioptix icon will launch it, where it can be seen its main menu, along with the two buttons for Exercise or Exit.

- Before starting exercises, it is extremely important to occlude the director eye, which is the eye with a much better visual acuity. A professional occlusive devices can be used or the eye it can be occluded by a handy method (for example, using a tissue). After that, the exercises for the amblyopic eye can be started;

- Pressing the "Exersează" button will access a new menu showing three exercises for eye recovery. The amblyopic subject can work with three exercises. They are numbered from 1 to 3 , on different levels of difficulty, from the most accessible to the most difficult.

- To begin with, exercise 1 is recommended, and the training time should be five minutes. To avoid "cheating", it can also call for additional people for support. In one training session, it is recommended to use the entire set of exercises.

- For a notable and positive result, daily exercise is continued and it is very important for the subject to follow the recovery protocol.

- It is also recommended to periodically submit to a specialist consultation during the use of this software.

\subsection{Application testing}

The application developed and presented above was tested on a group of students, two of them having amblyopia confirmed by specialized tests. 


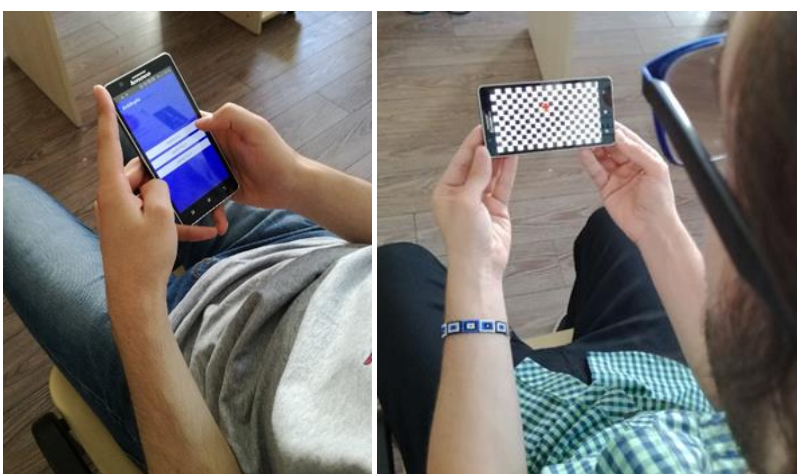

Fig. 8. Amblioptix testing

Application testing was conducted over three weeks, during which the subject used the app installed on your phone. The application was used for 5 minutes each day. Subjects with amblyopia began to work on difficulty levels from exercise 1 to exercise 3. Each exercise was used over a period of one week, a period of time necessary for the eye with amblyopia to get used to the work environment. The subjects felt better and easier to work with the lunch-time application, as opposed to the morning when they had difficulties in exercising.

After a period of six weeks, re-testing of amblyopia was performed by a specialist medical consultation and an obvious improvement in visual acuity was achieved by about $10 \%$.

\section{Conclusions}

The application proposed in this paper can become a very accessible tool for anyone who needs training for visual recovery. Its main advantage is that it can be installed on any device that runs using the Android operating system, which gives it great mobility. It takes a few minutes of use per day and can be used in school, office or any service that can provide a short break. For better results, the application should be accessed at different times of the day for visual application under different conditions of eye fatigue.

The development possibilities would consist in the fact that this app could reach the Play Store and so could be accessed by all children and young people, and not only, who have visual problems. As the application is specifically intended for those with amblyopia, it could be used every day by people with ocular problems to be able to perform recovery exercises in a simple and efficient way. Particularly, the application could also be used in optician and optometry offices, ophthalmic and paediatric clinics, as well as in educational institutions.

A real possibility of future development would be to design a more advanced application that could use access to the front camera phone to avoid cheating. Thus the front camera could detect eye movement and the subject would be warned whenever his eyes do not follow the target. Also a great advantage would be the possibility of timing, registration of the recovery program and the results obtained.

Still, performing these exercises is extremely important in visual recovery, and specialist medical advice is strictly recommended to be performed periodically.

Thanks to the students from the Optometry study program of Transilvania University of Brasov, and especially Alexandru Spataru, for the contribution made in this study.

\section{References}

1. M.I. Baritz, The $7^{\text {th }}$ International Conference on Computational Mechanics and Virtual Engineering, COMEC 2017, 182-187 (2017)

2. B. Braun, The 13th International Scientific Conference eLearning and Software for Education, ELSE 2017, 1, 500-505 (2017)

3. M.I. Baritz, The International Scientific Conference eLearning and Software for Education ELSE 2016 1, 498-502 (2016)

4. M.I. Baritz, Conference on Sustainable Energy CSE 2017, 720-729 (2017)

5. B. Braun, Bulletin of the Transylvania University of Brasov, 9 (58), 57-62 (2016)

6. P. Cernea, Treaty Ophthalmology, Medical Press, Bucharest (1997)

7. P. Cernea, F. Constantin, M. Aconiu, Strabismus, Medical Press (1981)

8. A. Pringle, S. Somerville, Mental Health Practice 17, 34-37 (2013)

9. D. Smite, C. Wohlin, T. Gorschek, R. Feldt, Journal of Empirical Software Engineering 15 (1), 91-118 (2010).

10. P. Kaur, S. Sharma, 2014 Recent Advances in Engineering and Computational Sciences (RAECS), Chandigarh, 1-5 (2014)

11. M.A. Anwar, D. Gangodkar, Communication Control and Intelligent Systems (CCIS) 2015, 120123 (2015)

12. A. Singh, S. Sharma, S. Singh, International Journal of Computer Applications (0975-8887), 5-8 (2016)

13. Android Official Website $\underline{\text { http://www.android.com/ (2018) }}$ 